\title{
TWO-DIMENSIONAL HILBERT TRANSFORMS ${ }^{1}$
}

\section{R. J. DUFFIN}

1. Introduction. An inspection of Chapter $\mathrm{V}$ of Introduction to the theory of Fourier integrals (Oxford, 1937) by E. C. Titchmarsh reveals that the following six topics have a natural relationship: Fourier transforms, Abel summability, Poisson's integral, Cauchy's integral, conjugate harmonic functions, and Hilbert transforms. The aim of this paper is to bring out the same relationships when the dimension is increased by one in each case. Thus of concern here are Fourier transforms of functions of two variables and harmonic functions of three variables.

The notion of a pair of conjugate harmonic functions in three variables is introduced by a suitable generalization of the Cauchy-Riemann equations. These conjugate functions are in general complex valued. The generalization of the Cauchy integral employed is due to A. C. Dixon [4] and involves quaternions. Proceeding by analogy with the classical theory one is led by these concepts to a pair of integral equations which are the analogs of the Hilbert transforms.

Various generalizations of Hilbert transforms and singular integrals have been given in the literature $[1 ; 3 ; 5 ; 6 ; 7 ; 9]$. Some of these generalizations have points of contact with the present developments.

2. Application of Fourier transforms. The Fourier transform formulae for the plane [2] are:

$$
\begin{aligned}
& U(x, y)=(2 \pi)^{-1} \int_{-\infty}^{\infty} \int_{-\infty}^{\infty} e^{i \bar{x} x+i \bar{y} y} u(\bar{x}, \bar{y}) d \bar{x} d \bar{y}, \\
& u(\bar{x}, \bar{y})=(2 \pi)^{-1} \int_{-\infty}^{\infty} \int_{-\infty}^{\infty} e^{-i \bar{x}-i \bar{y}} U(x, y) d x d y .
\end{aligned}
$$

It is convenient to abbreviate these relations as $U=T u$ and $u=T^{*} U$. To insure the validity of (1) and (2), attention is to be confined to functions of the class $L_{2}$. A function $u$ is in the class $L_{2}$ if its norm, $\int_{-\infty}^{\infty} \int_{-\infty}^{\infty}|u|^{2} d \bar{x} d \bar{y}$, is finite. Then if $u$ is of the class $L_{2}$, the integral in (1) converges in mean to a function $U$ of the class $L_{2}$. That is, if $U_{a}=\int_{-a}^{a} \int_{-a}^{a} e^{i x \bar{x}+i y \bar{y}} u(\bar{x}, \bar{y}) d \bar{x} d \bar{y}$, then the norm of $U-U_{a}$ vanishes as $a \rightarrow \infty$. The inverse transformation (2) holds in the same sense. According to Parseval's theorem, $u$ and $U$ have the same norm. The

Received by the editors May 31, 1956.

1 The preparation of this paper was sponsored by the Office of Ordnance Research, U. S. Army, Contract DA-36-061-ORD-490. 
transform of the product of two functions $u_{1}$ and $u_{2}$ is given by the convolution formula

$$
T\left(u_{1} u_{2}\right)=(2 \pi)^{-1} \int_{-\infty}^{\infty} \int_{-\infty}^{\infty} U_{1}(x-\bar{x}, y-\bar{y}) U_{2}(\bar{x}, \bar{y}) d \bar{x} d \bar{y} .
$$

A unitary transformation is a linear transformation which has an inverse and which preserves the norm. It follows from what has been said that the Fourier transform is unitary.

Let $u(x, y)$ be a given function of the class $L_{2}$ and let a function $U(x, y, z)$ be defined by

$$
U(x, y, z)=T\left(e^{-z r} u(\bar{x}, \bar{y})\right),
$$

where $r=\left(\bar{x}^{2}+\bar{y}^{2}\right)^{1 / 2}$. The integral on the right side of (4) is absolutely convergent. The limit of (4) as $z$ tends to zero gives an evaluation of the Fourier transform by what may be termed Abel summability. To investigate this summability method, the following special transform is needed

$$
T\left(e^{-z r}\right)=z\left(x^{2}+y^{2}+z^{2}\right)^{-3 / 2} .
$$

Applying the convolution formula to (4) and making use of (5) gives

$$
U(x, y, z)=\frac{1}{2 \pi} \int_{-\infty}^{\infty} \int_{-\infty}^{\infty} \frac{z U(\bar{x}, \bar{y}) d \bar{x} d \bar{y}}{\left[(x-\bar{x})^{2}+(y-\bar{y})^{2}+z^{2}\right]^{3 / 2}} .
$$

This is recognized as the Poisson integral formula for the half-space. From the theory of the Poisson integral it is known that $U(x, y, z)$ $\rightarrow U(x, y)$ almost everywhere as $z \rightarrow 0$. In particular this limit exists at points where $U(x, y)$ is continuous. Thus the Abel summability of a Fourier transform is consistent with the convergence in mean evaluation.

The Poisson integral gives a harmonic function, i.e., $\nabla^{2} U(x, y, z)$ $=0$. This can be seen directly from (4) because the "kernel" $e^{i x \bar{x}+i y \bar{y}-z r}$ is itself harmonic. This kernel serves to relate Fourier transforms and harmonic functions.

Let $U(x, y)$ be a function of the class $L_{2}$, then a unitary transformation is defined by

$$
V(x, y)=T\left[-i e^{-i \theta} T^{*} U(x, y)\right]
$$

where $r e^{i \theta}=\bar{x}+i \bar{y}$. The inverse transformation is

$$
U(x, y)=T\left[i e^{i \theta} T^{*} V(x, y)\right] .
$$

To prove this it is noted that $\left|-i e^{-i \theta}\right|=1$, so multiplication by $-i e^{-i \theta}$ does not change the norm. Thus $V$ is formed by a product of three 
norm preserving transformations applied to $U$, hence $V$ and $U$ have the same norm. It is apparent that (7) substituted in (8) yields the identity transformation. This proves that (7) is unitary. Relations (7) and (8) are here termed Hilbert transforms.

According to relation (4) a harmonic function $U(x, y, z)$ is associated with $U(x, y)$. Likewise a harmonic function $V(x, y, z)$ is associated with $V(x, y)$. Since $u(\bar{x}, \bar{y})=i e^{i \theta} v(\bar{x}, \bar{y})$, relation (4) gives

$$
V(x, y, z)=T\left[-i e^{-i \theta-z r} u(\bar{x}, \bar{y})\right]
$$

and

$$
U(x, y, z)=T\left[i e^{i \theta-z r} v(\bar{x}, \bar{y})\right] .
$$

These may be regarded as the Abel summability expressions for (7) and (8). As $z \rightarrow 0$ then $V(x, y, z) \rightarrow V(x, y)$ a.e. and $U(x, y, z) \rightarrow U(x, y)$ a.e.

The system of equations

$$
\begin{aligned}
& \frac{\partial U}{\partial z}+\frac{\partial V}{\partial x}+i \frac{\partial V}{\partial y}=0 \\
& \frac{\partial V}{\partial z}-\frac{\partial U}{\partial x}+i \frac{\partial U}{\partial y}=0
\end{aligned}
$$

is analogous to the Cauchy-Riemann system. In keeping with this analogy an ordered pair of functions $(U, V)$ which satisfies (11) will be termed a conjugate pair. It is now to be shown that $U$ and $V$ defined by (10) and (9) are conjugate. To do this (10) is replaced by the equivalent relation (4), and it follows that $\partial U / \partial x-i \partial U / \partial y$ $=T\left[i(\bar{x}-i \bar{y}) e^{-z r} u\right]$. It is seen from (9) that $\partial V / \partial z=T\left[i(\bar{x}-i \bar{y}) e^{-z r} u\right]$. This proves the second equation (11). The proof that the first equation is satisfied is analogous.

To apply the convolution theorem to (9) and (10), the following special transform is needed

$$
T\left(i e^{ \pm i \theta-z r}\right)=-(x \pm i y)\left(x^{2}+y^{2}+z^{2}\right)^{-3 / 2},
$$

Then (9) and (10) become

$$
\begin{aligned}
& V(x, y, z)=\frac{1}{2 \pi} \int_{-\infty}^{\infty} \int_{-\infty}^{\infty} \frac{[(x-\bar{x})-i(y-\bar{y})] U(\bar{x}, \bar{y}) d \bar{x} d \bar{y}}{\left[(x-\bar{x})^{2}+(y-\bar{y})^{2}+z^{2}\right]^{3 / 2}} \\
& U(x, y, z)=\frac{-1}{2 \pi} \int_{-\infty}^{\infty} \int_{-\infty}^{\infty} \frac{[(x-\bar{x})+i(y-\bar{y})] V(\bar{x}, \bar{y}) d \bar{x} d \bar{y}}{\left[(x-\bar{x})^{2}+(y-\bar{y})^{2}+z^{2}\right]^{3 / 2}}
\end{aligned}
$$

Formally allowing $z$ to approach $z$ ero gives the following more direct 
definition of the Hilbert transforms

$$
\begin{aligned}
& V(x, y)=\frac{1}{2 \pi} \int_{-\infty}^{\infty} \int_{-\infty}^{\infty} \frac{e^{-i \phi}}{R^{2}} U(\bar{x}, \bar{y}) d \bar{x} d \bar{y}, \\
& U(x, y)=\frac{-1}{2 \pi} \int_{-\infty}^{\infty} \int_{-\infty}^{\infty} \frac{e^{i \phi}}{R^{2}} V(\bar{x}, \bar{y}) d \bar{x} d \bar{y} .
\end{aligned}
$$

Here $R^{2}=(x-\bar{x})^{2}+(y-\bar{y})^{2}$ and $\tan \phi=(y-\bar{y}) /(x-\bar{x})$. The justification of this limiting operation from (13) to (15) for a function $U$ which satisfies a Hölder condition presents no difficulty; the proof is omitted. The theory of improper multiple integrals such as (15) and (16) is developed in references [3] and [7].

3. Regular quaternion functions. In the classical theory it is possible to derive the Hilbert transforms from Cauchy's integral. A three-dimensional generalization of the Cauchy integral formula will now be employed to derive the transforms (15) and (16).

A quaternion function is denoted by $F$. Then $F=W+i X+j Y+k Z$ where $W, X, Y$, and $Z$ are real functions of $x, y$, and $z$. The quaternion units $i, j$, and $k$ obey the rules $i^{2}=j^{2}=k^{2}=-1, i j=k=-j i, j k=i$ $=-k j$, and $k i=j=-i k$. Let $\nabla$ denote the quaternion operator $i \partial / \partial x+j \partial / \partial y+k \partial / \partial z$. A function $F$ is said to be regular in an open set if $\nabla F=0$ at each point. This condition expressed in terms of components is

$$
\begin{aligned}
& 0-\frac{\partial X}{\partial x}-\frac{\partial Y}{\partial y}-\frac{\partial Z}{\partial z}=0, \\
& \frac{\partial W}{\partial x}+0-\frac{\partial Y}{\partial z}+\frac{\partial Z}{\partial y}=0 \\
& \frac{\partial W}{\partial y}+\frac{\partial X}{\partial z}+0-\frac{\partial Z}{\partial x}=0, \\
& \frac{\partial W}{\partial z}-\frac{\partial X}{\partial y}+\frac{\partial Y}{\partial x}+0=0 .
\end{aligned}
$$

If the components of $F$ have second derivatives, then

$$
0=-\nabla \nabla F=\left(\partial^{2} / \partial x^{2}+\partial^{2} / \partial y^{2}+\partial^{2} / \partial z^{2}\right) F .
$$

Thus all the components of $F$ are harmonic.

This notion of regularity of a quaternion function was introduced by A. C. Dixon in 1904 [4]. He regarded equations (17) as a generalization of the Cauchy-Riemann equations. Generalizations of the Cauchy-Riemann equations have been studied by many writers. A 
survey of some of the older work in this field is given by Rainich [8], and some of the newer work is described in reference [10].

The conjugate quaternion to $F$ is $F^{*}=W-i X-j Y-k Z$. Let $G$ be another regular quaternion function. Then

$$
0=(\nabla G)^{*}=G^{*} \nabla^{*}=-G^{*} \nabla .
$$

The convention is adopted here that $\nabla$ can operate backward or forward unless blocked by a parenthesis sign. Thus

$$
G^{*} \nabla F=\left(G^{*} \nabla\right) F+G^{*}(\nabla F)=0 .
$$

Let $F$ and $G$ be regular in a region $R$ and on its boundary $S$. It is supposed that $R$ is of a type to which Green's theorem applies. Then integration by parts gives

$$
\iiint_{R} G^{*} \nabla F d x d y d z=\iint_{S} G^{*} n F d S
$$

where $n$ is the outward normal vector to the surface $S$. Making use of (18) gives

$$
\iint_{S} G^{*} n F d S=0
$$

This is an analog of the Cauchy theorem. Let $\rho$ be the vector from a fixed point $p$ to a variable point $q$. Let $G=\nabla|\rho|^{-1}=-\rho|\rho|^{-3}$. Then $\nabla G=\nabla^{2}|\rho|^{-1}=0$, so $G$ is regular except at the point $p$. If $p$ is an interior point of $R$, then

$$
F(p)=\frac{1}{4 \pi} \iint_{S} \frac{-\rho n F(q)}{|\rho|^{3}} d S .
$$

This follows from (19) by excluding from the region a small sphere centered at $p$. Formula (20) is Dixon's analog of the Cauchy integral formula.

Let $F$ be regular in the half-space $z \geq 0$, and suppose that as $p$ becomes infinite $F(p) \rightarrow 0$ uniformly in this half-space. Formula (20) may be applied to a hemispherical region $R$ which is the intersection of the sphere of radius $b$ centered at the origin and the half-space $z \geq 0$. With $p$ fixed, $b$ is allowed to approach infinity. Then the part of the integral over the spherical surface vanishes, so if $p$ has coordinates $(x, y, z)$ with $z>0$ and $q$ has coordinates $(\bar{x}, \bar{y}, 0)$,

$$
F(p)=\frac{1}{4 \pi} \int_{-\infty}^{\infty} \int_{-\infty}^{\infty} \frac{\rho k F(q)}{|\rho|^{3}} d \bar{x} d \bar{y} .
$$


If $z<0$, then it follows from (19) that the integral in (21) vanishes.

To relate the notions of conjugate functions with quaternion functions, we write

$$
F=(W+k Z)+j(Y+k X)=U+j V .
$$

Thus a quaternion may be regarded as an ordered pair of complex numbers $(U, V)$. If $F_{1}$ and $F_{2}$ denote two quaternions, then

$$
\begin{aligned}
F_{1} F_{2} & =U_{1} U_{2}+j V_{1} j V_{2}+j V_{1} U_{2}+U_{1} j V_{2} \\
& =\left(U_{1} U_{2}-V_{1}^{*} V_{2}\right)+j\left(V_{1} U_{2}+U_{1}^{*} V_{2}\right) .
\end{aligned}
$$

Then the formula for the product of two quaternions may be written

$$
\left(U_{1}, V_{1}\right)\left(U_{2}, V_{2}\right)=\left(U_{1} U_{2}-V_{1}^{*} V_{2}, V_{1} U_{2}+U_{1}^{*} V_{2}\right) \text {. }
$$

(It is of interest to note that relation (22) could serve as a definition of quaternions.)

The condition that $F$ be a regular function can now be written

$$
(k \partial / \partial z, \partial / \partial y+k \partial / \partial x)(U, V)=0 .
$$

This is seen to be the same as the system of equations (11) with $k$ replacing $i$. Thus a regular quaternion function is equivalent to a conjugate pair.

Returning to the Cauchy type formula (21), let $z^{\prime}=-z$ and let $\rho^{\prime}$ denote $\rho$ with $z$ replaced by $z^{\prime}$. Then $\left|\rho^{\prime}\right|=|\rho|$ and $\rho+\rho^{\prime}=2 i(\bar{x}-x)$ $+2 j(\bar{y}-y)$, so $(21)$ gives

$$
F(p)=\frac{1}{2 \pi} \int_{-\infty}^{\infty} \int_{-\infty}^{\infty} \frac{j(x-\bar{x})-i(y-\bar{y})}{|\rho|^{3}} F(q) d \bar{x} d \bar{y} .
$$

Expressing this relation in terms of complex components gives

$$
(U, V)=\frac{1}{2 \pi} \int_{-\infty}^{\infty} \int_{-\infty}^{\infty} \frac{[0,(x-\bar{x})-k(y-\bar{y})]}{|\rho|^{3}}(U, V) d \bar{x} d \bar{y} .
$$

This relation is seen to be equivalent to relations (13) and (14). Allowing $z$ to approach zero gives the Hilbert transforms (15) and (16). The limiting operation is justified because by hypothesis $U$ and $V$ have derivatives, so the integrals in (15) and (16) are convergent.

It is of interest to take $G=F$ in formula (19). The region of integration is again taken to be the hemisphere. It is supposed that $F$ vanishes sufficiently rapidly at infinity so that the integral over the spherical part of the surface vanishes as $b \rightarrow \infty$. Then in the limit $\int_{-\infty}^{\infty} \int_{-\infty}^{\infty} F^{*} k F d \bar{x} d \bar{y}=0$. Now it may be verified that 


$$
F^{*} k F k=V^{*} V-U^{*} U+2 j V U \text {. }
$$

Thus it follows that $\iint|V|{ }^{2} d \bar{x} d \bar{y}=\iint|U|^{2} d \bar{x} d \bar{y}$ and $\iint U V d \bar{x} d \bar{y}=0$. The first relation does not contribute anything new, but the second relation gives an orthogonality property of the Hilbert transform. This property may also be proved by the method of Fourier transforms.

Consider the class of regular quaternion functions such that $\int_{-\infty}^{\infty} \int_{-\infty}^{\infty}|F(x, y, c)|^{p} d x d y$ is uniformly bounded for all $c>0$. In the book cited, Titchmarsh presents an interesting theory of the analogous class of functions of a complex variable; presumably, features of this theory could be extended to the regular quaternion functions.

The author wishes to acknowledge helpful discussion concerning the topics in this paper with Dr. Thomas A. Elkins.

\section{REFERENCES}

1. Edward Block, Kernel functions and class $L^{2}$, Proc. Amer. Math. Soc. vol. 4 (1953) pp. 110-117.

2. S. Bochner and K. Chandrasekharan, Fourier transforms, Princeton University Press, 1949.

3. A. P. Calderon and A. Zygmund, On the existence of certain singular integrals, Acta Math. vol. 88 (1952) pp. 88-139.

4. A. C. Dixon, On the Newtonian potential, Quart. J. Math. Oxford Ser. vol. 35 (1904) pp. 283-296.

5. R. J. Duffin, Basic properties of discrete analytic functions, Duke Math. J. vol. 23 (1956) pp. 335-364.

6. J. Horváth, Sur les fonctions conjugées à plusieurs variables, Koninklijke Nederlandsche Akademie van Wetenschappen vol. 15 (1953) pp. 17-29.

7. S. G. Mihlin, Singular integral equations, Amer. Math. Soc. Translations, No. 24, 1950.

8. G. Y. Rainich, Analytic functions and mathematical physics, Bull. Amer. Math. Soc. vol. 37, 1931 pp. 689-714.

9. O. A. Varsavsky, Sobre la transformation de Hilbert, Revista de la Unión Matemática Argentina vol. 14 (1949) pp. 20-37.

10. W. T. Martin, S. S. Chern, and Oscar Zariski, Scientific report on the Second Summer Institute, Several Complex Variables, Bull. Amer. Math. Soc. vol. 62 (1956), Part I, §5.

Carnegie Institute of Technology 